\title{
Evaluation of Vibration Properties of Three-Dimensional, Four-Directional Braided Composites*
}

\author{
By Jing Pu, ${ }^{1), 2)}$ Apeng Dong, ${ }^{1)}$ Shu $\mathrm{LI}^{1)}$ and Zhixin ZHAN ${ }^{1)}$ \\ ${ }^{1)}$ School of Aeronautic Science and Engineering, Beihang University, Beijing 100191, China \\ ${ }^{2)}$ Department of Mechanical, Material and Manufacturing Engineering, University of Nottingham, Nottingham NG7 2RD, United Kingdom
}

(Received February 20th, 2017)

\begin{abstract}
This paper investigates the relationship between natural frequencies and different braiding parameters: the braiding angles and the fiber volume fractions for three-dimensional, four-directional (3D4d) braided composites. A systematic experiment was set up and conducted, and a corresponding finite element model was built to analyze the experimental results. Since the rate of change among the first three orders of natural frequencies for changing braiding parameters are strongly monotonous, only the first order is analyzed. Based on experimental data, the relationship between the natural frequencies of the first order and the two types of braiding parameters are compared and discussed. The findings suggest that the natural frequency of this material and its 3D4d structure can be designable.
\end{abstract}

Key Words: 3D4d Braided Composites, Braiding Angles, Fiber Volume Fractions, Natural Frequencies

\section{Nomenclature}

$\begin{array}{cll}1,2,3 & : & \text { ply material axes respective properties } \\ A & : & \text { half-circumference of the unit cell } \\ D & : & \text { distance between two skew lines of } \\ & \text { braiding yarns } \\ E & : & \text { elastic properties matrix as defined by } \\ & \text { subscripts; modulus, as defined by sub- } \\ & \text { scripts }\end{array}$

(C) 2017 The Japan Society for Aeronautical and Space Sciences ${ }^{*}$ Presented at the 2016 Asia-Pacific International Symposium on Aerospace Technology (APISAT-2016), Oct. 25-27, 2016, Toyama, Japan

\section{Introduction}

Recently 3D braided composites are gaining increasing importance in industrial applications. Examples of applications of 3D braided composites are propeller blades, propulsion shafts, propellers, truss section decking, landing pads, car bodies, chassis, drive shafts, biomedical devices etc. The technology of manufacturing $3 \mathrm{D}$ composites is developed based on that of $2 \mathrm{D}$ composites. Researchers have found that the strength of the $3 \mathrm{D}$ composites is the same or slightly higher than an equivalent 2D composite material. The impact resistance, compression after impact, and delamination control are significantly improved with a $3 \mathrm{D}$ composite without reducing other mechanical properties. ${ }^{1)}$ In this case, the composite part consist of plies, just as with $2 \mathrm{D}$ fabric, the basic $3 \mathrm{D}$ preform has the fibers oriented primarily in two orthogonal directions. ${ }^{2)}$ Three-dimensional composites can be created from a 3D weaving process, a 3D braiding process, or a 3D deposit of short fibers. Among these three, 3D braided fabrics technology is an extension of the wellestablished 2D braiding technology wherein the fabric is constructed by the intertwining of two or more yarn systems to form an integral structure. ${ }^{3)}$ Among the different types of 3D braiders applied in manufacturing, two types, which are horn gear and track-and-column types, are the main types. It is also possible to produce $3 \mathrm{D}$ solid braids with a variety of cross-sections. ${ }^{4,5}$ ) A significant amount of research has been done on the elastic properties of 3D braided composites. For instance, Cai et al. ${ }^{6}$ investigated the stiffness and damping of the yarn with an rectangular cross-section of three-dimensional four-directional (3D4d) braided composites. However, little attention has been paid to the vibration properties of this type of composites. Cawley and Adams ${ }^{7)}$ were the first to use the change in frequency for vibration modes to detect structural damage in composites. Li et al. ${ }^{8)}$ conducted a thorough study on 3D4d braided composites using the free vibration of cantilever beams. It has been demonstrated that the damping properties of composites are improved when the braiding angle increases or the fiber volume fraction decreases. Pei and co-workers ${ }^{9,10)}$ explored the influ- 
ence of the microstructural effect and braiding parameters on the dynamic behavior of 3D4d braided composites using a discrete beam model.

This work investigates the vibration properties of 3D4d braided composites with different braiding parameters: the braiding angles and fiber volume fractions. The purpose of this investigation is to explore the effect of varying of the braiding parameters on the vibration behavior according to the 3D4d braiding structure. Based on experimental data, the relationship between the natural frequencies and two braiding parameters can be compared and discussed.

In this paper, Section 2. gives a basic description of how the braided composite specimens were manufactured; Section 3. introduces the specimen, method and equipment used in this experiment, after which the experimental results are presented; Section 4. includes the model description, finite element modeling, and a comparison of the computational and experimental results; the last section, Section 5., provides a summary of what was found in the experiment and finite element analysis (FEA).

\section{Braiding Process}

3D4d braided composites are produced using a 4-step process, which is the most common way to produce braided composites. Each of the four steps is implemented by a single machine cycle. In the first machine step, alternate rows of yarn are moved on through the opposite direction of $x$-directions. In the second machine step, likewise, alternate columns of yarn are moved on through the opposite direction of $y$-directions. The third and fourth machine steps are in reverse directions of the first two steps, respectively. A periodic topological structure is formed as one braiding cycle is accomplished. Figure 1 schematically depicts 4 -step processed 3D4d braided composites.

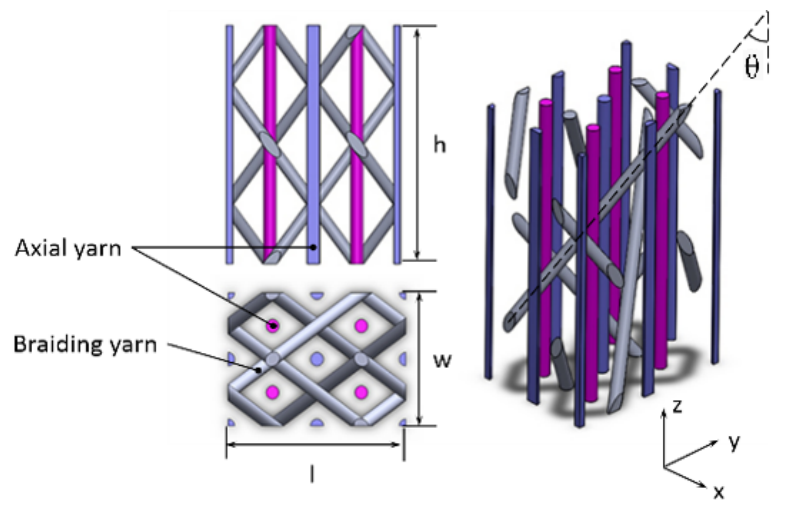

Fig. 1. 4-step processed 3D4d braided composites.

\section{Experiment}

\subsection{Specimen, method and equipment}

All preforms were produced by Beijing 3D Braided Co., Ltd. with different braiding angles and fiber volume fractions. These preforms were consolidated with epoxy resin into the final sample using Resin Transfer Molding (RTM). The fiber and matrix are T700-12K and EpoTech4237 epoxy resin. Their mechanical properties are listed in Table 1.
The size of the specimen is ensured to be $250 \mathrm{~mm}$ (length) $\times 25 \mathrm{~mm}$ (width) $\times 5 \mathrm{~mm}$ (thickness) using a specific mold equipped with two parallelized flow passages. Using $5 \mathrm{~mm}$ as support to create the cantilevered condition, the effective dimensions of the cantilever were reduced to $200 \mathrm{~mm} \times 25 \mathrm{~mm}$ $\times 5 \mathrm{~mm}$. To assist the experiment, a normalized rectangular Cartesian coordinate system with the surface-fitting method was used. During the experiment, a Kistler (8202A10) accelerometer was adhesively mounted near the free-end of the sample. The specimen was excited by the impulse hammer near the fixed end. The frequency range excited was controlled by the mass of the metal hammer head. The experiment setup is shown in Figure 2.

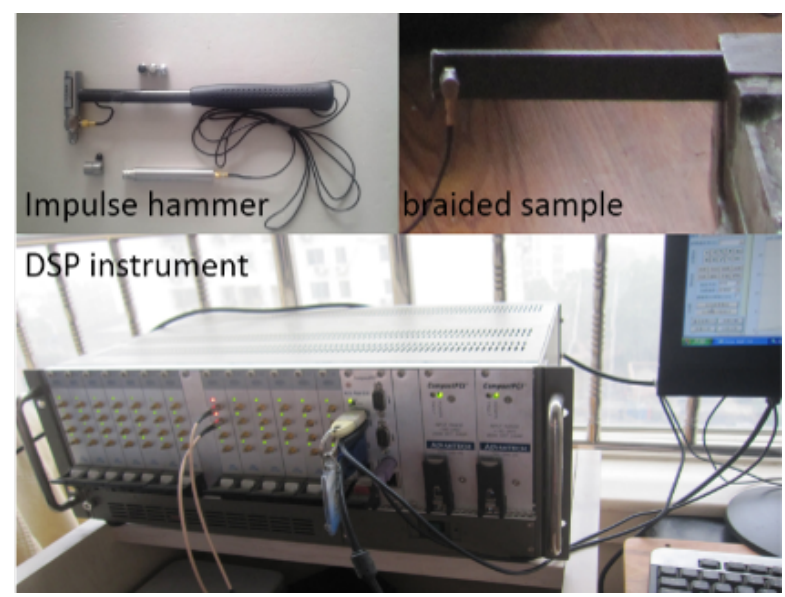

Fig. 2. Experimental equipment.

\subsection{Experimental results}

In order to investigate the relationship between braiding parameters and natural frequencies, the braiding parameters used and the experimentally found natural frequencies for each of the 12 specimens are listed in Table 2 . In this table, $\theta$ refers to the braiding angle, while $V_{\mathrm{f}}$ means the fiber volume fraction. As can be seen in Figure 3, the results show a monotonous rate between the first three orders of frequency. In order to remain brief and avoid a repetitive and unnecessary lengthy treatise of the results, only the first order of the frequency case related with the braiding parameters is discussed.

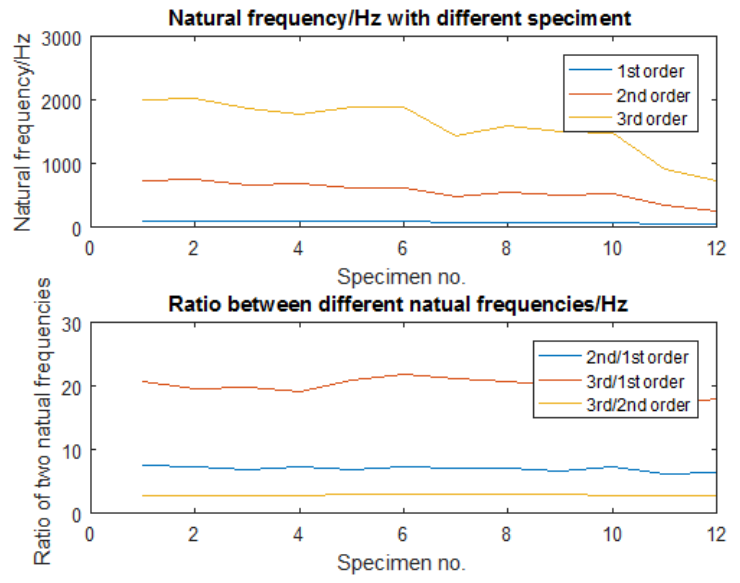

Fig. 3. Frequency ratio analysis with different orders.

Figure 4 shows a $3 \mathrm{D}$ graph of the $1^{\text {st }}$ order natural frequencies for different braiding parameters. 
Table 1. Mechanical properties of fibers and epoxy resin used in the experiment.

\begin{tabular}{llllll}
\hline Material & $E_{1} / G_{p a}$ & $E_{2} / G_{p a}$ & $G_{13} / G_{p a}$ & $G_{23} / G_{p a}$ & $v_{12}$ \\
\hline T700-12K & 230 & 40 & 24 & 14.3 & 0.26 \\
EpoTech4237 & 4.8 & - & - & - & 0.3 \\
\hline
\end{tabular}

Table 2. Braiding parameters and natural frequencies obtained during experiment.

\begin{tabular}{llllll}
\hline Specimen & \multicolumn{4}{l}{ Braiding parameters } & \multicolumn{4}{l}{ Natural frequency/Hz } & $2^{\text {nd }}$ \\
\hline no & $\theta /^{0}$ & $V_{f} / \%$ & $1^{\text {st }}$ & 725.7 & 2005.1 \\
\hline 1 & 16.5 & 46.6 & 97.2 & 743.6 & 2035.0 \\
\hline 2 & 19.8 & 49.9 & 103.6 & 655.0 & 1865.0 \\
\hline 3 & 22.0 & 50.1 & 94.6 & 674.9 & 1785.0 \\
\hline 4 & 27.9 & 52.9 & 92.9 & 619.5 & 1881.3 \\
\hline 5 & 28.4 & 47.8 & 89.9 & 625.0 & 1895.0 \\
\hline 6 & 29.4 & 43.8 & 86.5 & 481.8 & 1443.8 \\
\hline 7 & 34.1 & 51.8 & 68.5 & 543.7 & 1581.3 \\
\hline 8 & 36.2 & 50.0 & 76.7 & 493.5 & 1493.8 \\
\hline 10 & 38.0 & 48.5 & 75.0 & 527.5 & 1472.5 \\
\hline 11 & 40.3 & 48.1 & 71.3 & 331.3 & 919.0 \\
\hline 12 & 45.9 & 39.9 & 52.0 & 256.7 & 731.1 \\
\hline
\end{tabular}

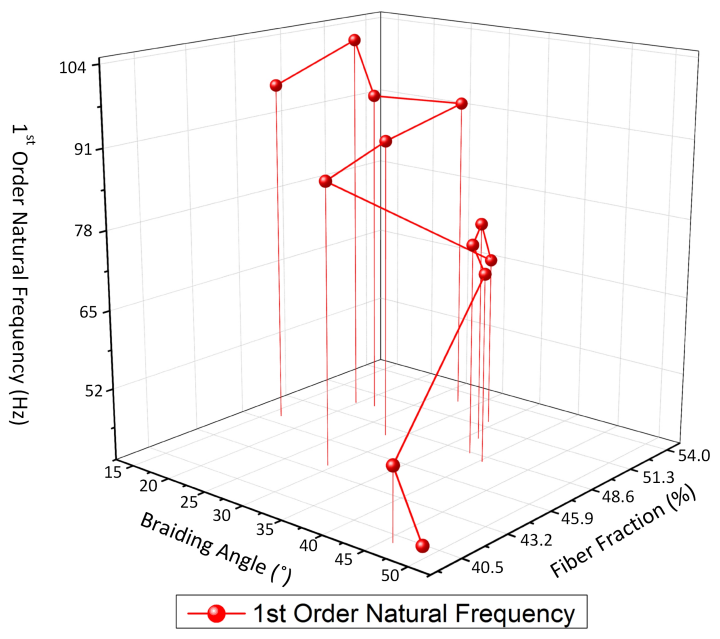

Fig. 4. First-order natural frequency experimental data.

\section{Finite Element Analysis and Results}

The major purpose of using finite element analysis (FEA) in this study is to predict the vibration properties of braided composites. Averaged anisotropic material properties are used in a 3D finite element model of a cantilevered beam to extract the frequencies. In order to obtain these averaged anisotropic material properties, however, a so-called unit cell must be built. Firstly, assumptions of the geometrical characteristics for the 3D braided fabric structural model are made. Secondly, reasonable (i.e., approaching those in the real world) boundary conditions are set. Finally, with the data of the unit cell, the equivalent mechanical elastic properties corresponding to those studied experimentally are obtained for 12 cases. Based on the static analysis of the unit cell, the macroscopic material properties are defined in the FEA, and then the harmonic, model and other dynamic analyses are carried out for the entire component.

\subsection{Unit cell modelling approach}

The solid model is directly constructed using the finite element modeling method. Based on the hypothesis to reduce

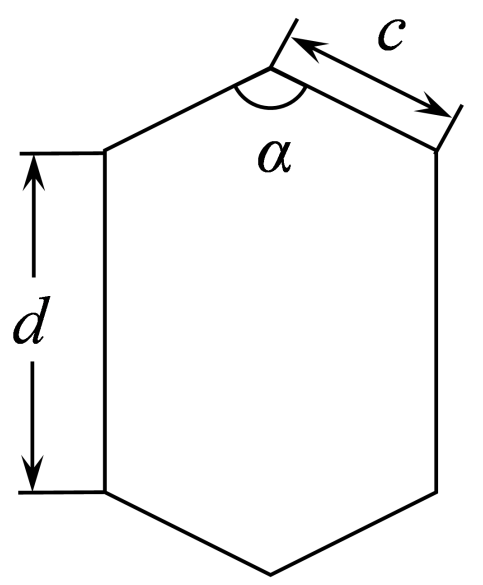

Fig. 5. Sectional view of knitting yarn interface.

the complexity of the geometric model, the material properties and unit cell geometry are established. Subsequently, the four local coordinate systems are set thereafter, enabling four directional yarns to be generated cyclically. Lastly, after static analyses, the unit cell structural properties are extracted.

\subsubsection{The hypothesis of the geometric model}

Under the assumption that the composite material is homogeneous, the following further assumptions are made on the geometric model:

1. The quality of the braiding process is constant, at least to a certain degree.

2. Even when adjacent yarns are touching, the cross-section of the braiding yarn is kept as hexagon. This hexagon is as shown in Figure 5. Here, $c$ refers to the cross-section cable length of the braiding yarn, $d$ refers to the cross-section straight length of the braiding yarn, and $\alpha$ is the angle between the adjacent cable lengths.

3. Adjacent fiber bundles may contact each other, all fiber bundles have the same characteristics, and the fiber bundle is not distorted. 


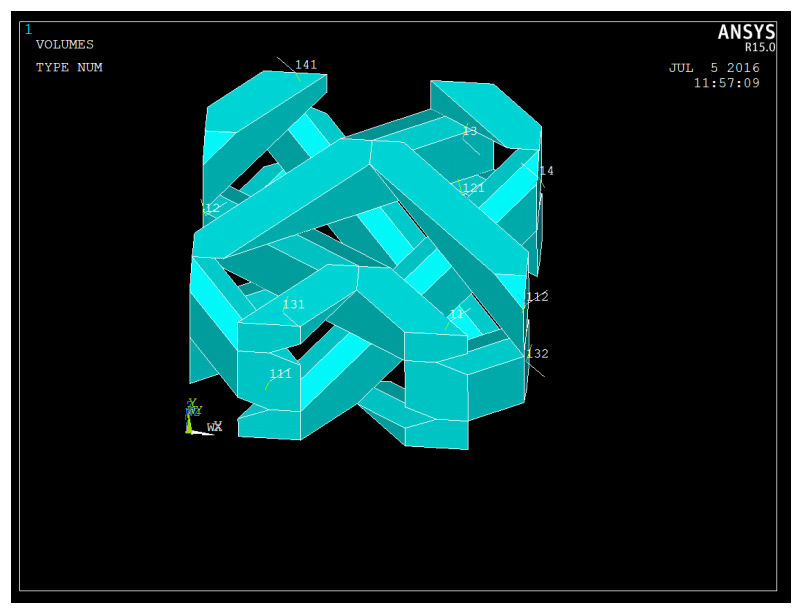

Fig. 6. Geometric model of the 3D braided fabric in the 3D4d unit cell used for FEA.

\subsubsection{Mathematics description of unit cell braiding geom- etry}

The 3D braided fabric is a periodic structure consisting of continuous fibers. FEA is usually not carried out using microstructures at the product or component level. Instead, a representative unit cell is selected and analyzed to extract the anisotropic material properties and these are used in subsequent analysis at the macroscopic level. For the four-directional structure, based on the geometric model assumptions, the internal yarn structure of the unit cell is established. The contact relationship between the yarns in the four-directional braiding structure is mainly the longitudinal contact between two knitting yarns. Figure 6 shows the geometry of the four-direction braided fabric in the unit cell.

In this model, the unit cell size is supposed to be $2 A \times 2 A \times$ $h$, where lengths, $2 A$ and $h$ are conveniently taken. Then the braiding angle can be obtained as Eq. (1):

$$
\tan \theta=\frac{2 \sqrt{2} A}{h},
$$

The relationship between $c, \alpha$, and $A$ is defined in Eq. (2):

$$
2 \sqrt{2} c \sin \frac{\alpha}{2}=A
$$

The center line between two braiding yarns is called the skew line. Parameter $D$ refers to the distance between these skew lines. The relationship between two adjacent braiding yarns is given in Eq. (3) is as follows:

$$
2\left(\frac{d}{2}+c \cos \frac{\alpha}{2}\right) \sin \frac{\alpha}{2}=D
$$

From the distance equation between the two skew lines, $D$ is also obtained as Eq. (4):

$$
D=\frac{A}{\sqrt{\frac{1}{2} \tan ^{2} \theta+1}},
$$

Using the geometric relationships, $\alpha$ is obtained as Eq. (5):

$$
\alpha=2 \arcsin \frac{1}{\sqrt{1+\cos ^{2} \theta}},
$$

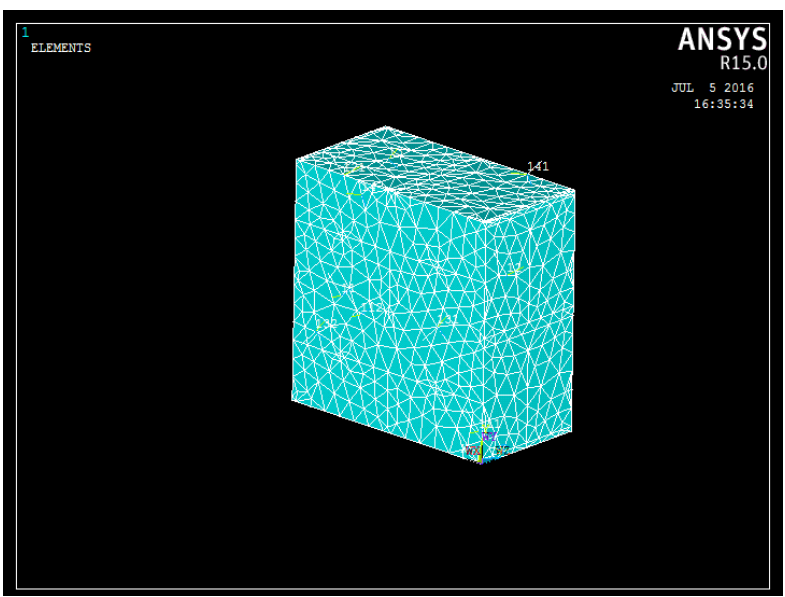

Fig. 7. Mesh of the 3D4d unit cell.

The cross-section area of the braiding yarn is determined using Eq. (6):

$$
S_{4}=c^{2} \sin \alpha+2 d c \sin \frac{\alpha}{2}=\frac{N \pi\left(\frac{\varphi}{2}\right)^{2}}{\varepsilon_{4}},
$$

Where $N$ is the braiding yarn's number of fibers, $\varphi$ is the fiber diameter of the braiding yarn, and in this work it is assumed to be $7 \mu \mathrm{m} . \varepsilon_{4}$ is the cross-section packing fraction of the braiding yarn, in this model, $\varepsilon_{4}$ is considered to be $\pi / 4$. Finally the fiber volume fraction $V_{\mathrm{f}}$ is obtained as Eq. (7):

$$
V_{\mathrm{f}}=\frac{V_{\mathrm{k}}}{V_{c}}=\frac{4 S_{4} \frac{h}{\cos \theta} \varepsilon_{4}}{(2 A)^{2} h}=\frac{N \pi\left(\frac{\varphi}{2}\right)^{2} \frac{1}{\cos \theta}}{A^{2}},
$$

In this equation, $V_{\mathrm{k}}$ is the volume of unit cell yarn. $V_{\mathrm{c}}$ is the volume of the unit cell. According to the topological structure, the coordinates of the so-called 'key points' used in the FE model can be calculated. Since these coordinates are related to the geometry of the unit cell, the model of the latter can now fully be described.

\subsection{Finite element modeling}

The FEA for the work presented in this paper was carried out using the commercially available FE software package ANSYS R15.0. ${ }^{11)}$ The geometry of the unit cell is discretized, and the material properties and boundary conditions are assigned.

\subsubsection{Grid division}

A 3D structural, tetrahedral element, SOLID45, is used in the FEA. The fiber bundle and fiber bundle contact surface, and fiber bundle and interface of the matrix are carefully used to ensure the continuity of displacement. The cell body is meshed with tetrahedral elements for cyclic boundary conditions to ensure the continuity and periodicity of the mechanical properties of the unit cell under stress, and the meshes on the opposing surfaces are identical in each direction. This is achieved by copying the mesh pattern from one side to the opposing side, thus forming a periodic mesh structure in the whole cell. The mesh is shown in Figure 7.

\subsubsection{Material properties}

The 3D braided composites are composed of two kinds of materials, which are the fibers and the epoxy resin matrix. Due to the different spatial orientations of the yarn, 
the geometric model is set up in the yarn local coordinate system. The coordinate system of each type of yarn unit is set up with the material properties. The elastic properties of the fiber and resin matrix are the same as those found in Table 1.

\subsubsection{Boundary condition}

The main objective to anayze the unit cell is to extract the mechanical properties from the periodic spatial structure. To obtain the averaged mechanical properties of the unit cell, periodic boundary conditions are imposed on the cell. The detail is as follows: $z$-direction is defined as molding direction:

Calculation of the elastic moduli: a displacement is applied at $z=0$ in the negative $z$-direction and at $z=h$ in the positive $z$-direction. The same method is used for the $x$-direction and $y$-direction. After that, the calculations of elastic modulus $E_{x}$ and $E_{y}$ are processed. Calculation of shear modulus $G_{x y}$ : a displacement is imposed in the $y$-direction by traversing the nodes of the $x=2 A$ surface. The deformation trend at $x=0$ is in the opposite $y$-direction. The same approach is used to obtain the material properties in the $x$-direction and $y$-direction. Additionally the calculations of shear moduli $G_{x z}$ and $G_{y z}$ are processed. Poisson's ratio is also obtained for the elastic properties.

The average stress and strain are calculated by the finite element software, and the equivalent, averaged directional elastic moduli of 3D braided composites are obtained. The equivalent elastic properties of composite materials can then be calculated using Eq. (8) to Eq. (12):

$$
\begin{gathered}
E_{1}=\varepsilon E_{\mathrm{f} 11}+(1-\varepsilon) E_{\mathrm{m}}, \\
E_{2}=E_{3}=-\frac{E_{\mathrm{m}}}{1-\sqrt{\varepsilon}\left(1-\frac{E_{\mathrm{m}}}{E_{\mathrm{f} 12}}\right)}, \\
G_{12}=G_{13}=\frac{G_{\mathrm{m}}}{1-\sqrt{\varepsilon}\left(1-\frac{G_{\mathrm{m}}}{G_{f 12}}\right)}, \\
G_{23}=\frac{G_{\mathrm{m}}}{1-\sqrt{\varepsilon}\left(1-\frac{G_{\mathrm{m}}}{G_{\mathrm{f} 23}}\right)}, \\
\mu_{12}=\mu_{13}=V_{\mathrm{f}} \mu_{\mathrm{f} 12}+(1-\varepsilon) \mu_{\mathrm{m}},
\end{gathered}
$$

\subsection{Macroscopic analysis of specimen model}

The finite element numerical result is compared to the experimental results, in order to verify the accuracy of the finite element model. The frequency analysis macroscopic model is described as follow: the averaged, anisotropic material properties found in the analysis of the unit cell are now used in the macroscopic FEA of the entire beam. They consist of SOLID45 linear 3D structural brick elements; the beam geometry and mesh are shown in Figure 8. It can be seen from this figure that the cantilever boundary condition is imposed on the side of the beam.

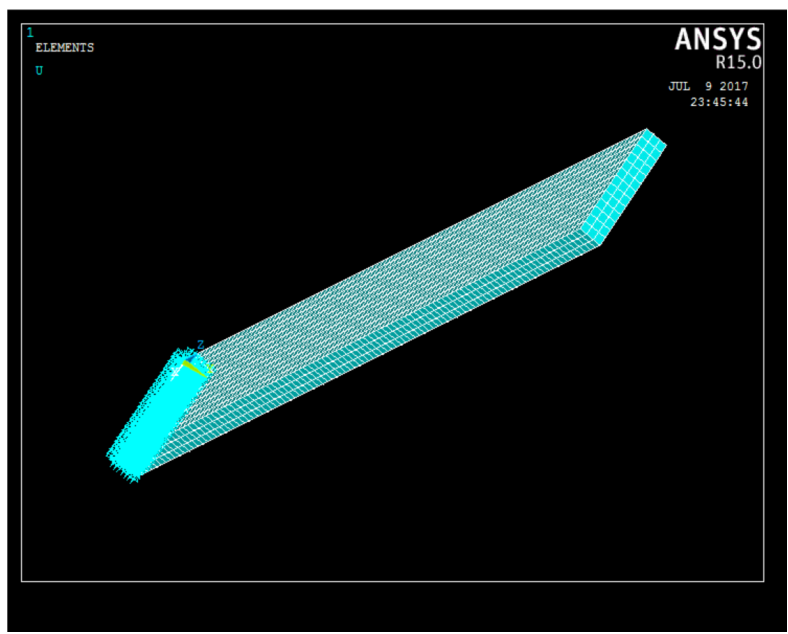

Fig. 8. Mesh and boundary conditions of the beam geometry.

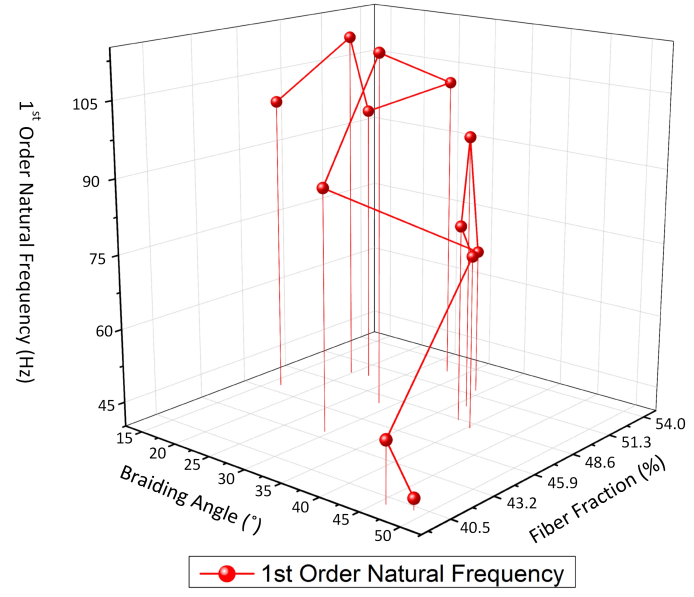

Fig. 9. 1st-order natural frequency $(\mathrm{Hz})$ numerical values of braiding angle and fiber fraction.

\subsection{Comparison of computational and experimental re- sults}

Standard frequency extraction analysis is applied to obtain the frequencies. Table 3 gives the results of the first-order natural frequency.

Figure 9 shows the data distribution of numerical results among the first-order natural frequency, braiding angles and fiber volume fractions. Figure 10 is the comparison between the experimental data and numerical results, and Figure 11 is the error between them.

From these figures, we can easily see that the predicted frequency using the FE model is generally higher than the experimental value due to the discretization. Some of the errors approach $20 \%$. It should also be noted that the prediction for specimens with higher natural frequencies shows a larger deviation. However, generally speaking, the finite element calculation results are similar to the experimental data. The reasons for the larger errors are possibly:

1. During the experiment, it is impossible to make a specimen with ideal rigid support. Therefore, this led to a testing frequency slightly lower than the natural frequency.

2. The assumption of the single-cell topology model is too ideal during the process of elastics property calculation. However, it is impossible to achieve ideal 3D braided com- 
Table 3. 3D4d numerical solution error.

\begin{tabular}{cccc}
\hline \multirow{2}{*}{ Specimen No. } & \multicolumn{2}{c}{ Natural frequency/Hz $1^{\text {st }}$} & \multirow{2}{*}{ Error/\% } \\
& Experimental result & Numerical result & \\
\hline 1 & 97.2 & 100.3 & 3 \\
\hline 2 & 103.6 & 112.1 & 8 \\
\hline 3 & 94.6 & 97.3 & 3 \\
\hline 4 & 92.9 & 102.7 & 10 \\
\hline 5 & 89.9 & 112.0 & 20 \\
\hline 6 & 86.5 & 89.0 & 3 \\
\hline 7 & 68.5 & 70.0 & 2 \\
\hline 8 & 76.7 & 95.8 & 20 \\
\hline 9 & 75.0 & 79.9 & 6 \\
\hline 10 & 71.3 & 75.1 & 5 \\
\hline 11 & 52.0 & 52.3 & 1 \\
\hline 12 & 40.5 & 42.3 & 4 \\
\hline
\end{tabular}

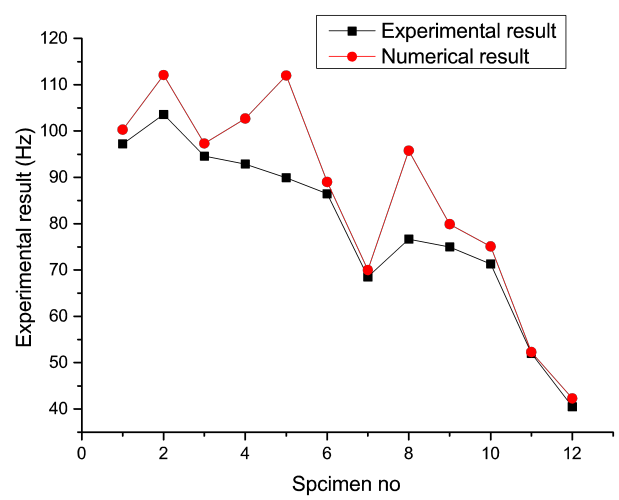

Fig. 10. The data distribution of experimental data and numerical results.

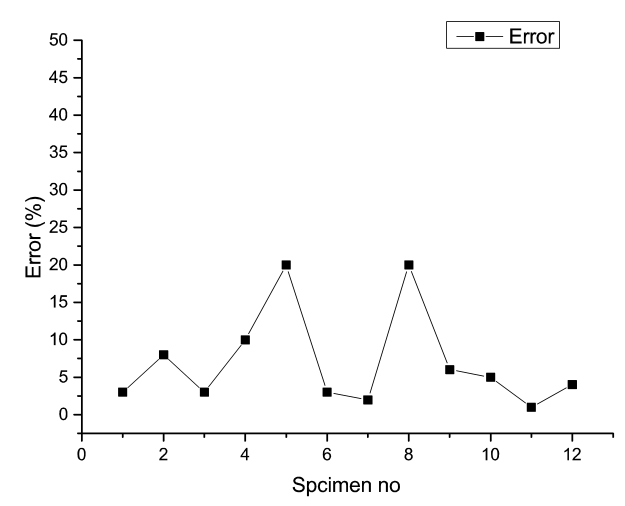

Fig. 11. Error between experimental data and numerical results.

posites in the process of actual braiding and solidification. The elastics constants calculated with the model lead to a higher natural frequency, which in turn, caused the high computational frequencies.

3. The effect of the damping on the natural frequencies is not considered when calculating natural frequencies. Even though the damping of isotropic material has little effect on the natural frequency, it cannot be neglected in 3D braided composites.

4. The mass of the acceleration sensor is comparatively large, which is not considered in FEA, and which also leads to error.

\section{Conclusions}

In order to clarify the relationship between braiding parameters and vibration behavior, the braiding angles, fiber volume fractions and first-order natural frequencies were extracted. This was done by means of a two-step finite element analysis: firstly a unit cell was established to model the mechanical properties of the micro-structure. The averaged anisotropic material properties were extracted and subsequently used in the second analysis to extract the natural frequencies of a beam structure. In addition, specimens with the same beam type geometry were manufactured and their natural frequencies were obtained experimentally to verify the accuracy of the finite element models. This study reveals that there is a monotonous relationship among the first three order natural frequencies. The natural frequency of a 3D4d braided composite increases when the braiding angle is reduced and decreases when the braiding angle increases. The natural frequency of 3D4d braided composites increases when the fiber volume fraction increases and decreases when the the fiber volume fraction decreases. In the 3D4d structure, the braiding angle plays the most influential role in affecting natural frequencies in this material. It presents a nearly monotonic relationship with natural frequencies. Since changing the braiding angle changes the stiffness, and consequently changes the frequency, the findings have great significance for further dynamic research of this material. This suggests that the natural frequency of this material and its 3D4d structure can be designed easily. The braiding parameters, including braiding angle and fiber volume fractions, should be considered as two crucial factors when designed.

\section{Acknowledgements}

This work was funded through the Industry-University Cooperation Project of the Aviation Industry Corporation of China, the support of which the authors greatly appreciated. In addition, the first author also wishes to thank Dr. Otto Jan Bakker for proofreading this manuscript. 


\section{References}

1) Mahmood, A., Wang X. and Zhou C.: Elastic Analysis of 3D Woven Orthogonal Composite, Grey Systems Theory and Application, 1, 3 (2011), pp. 228-239.

2) Michael, M. and Jonathan, G.: Overview of Recent Developments in 3D Structures, Albany Engineered Composites (AEC), Rochester, 2013, pp. 1-10.

3) Ko, F. K. : 3-D Textile Reinforcements in Composite Materials, In: 3-D Textile Reinforcements in Composite Materials, ed. Miravete, A., 1999, pp. 9-42.

4) Tada, M., Uozumi, T, Nakai, A. and Hamada, H.: Structure and Machine Braiding Procedure of Coupled Square Braids with Various Cross Sections, Composites Part A; 32, 10 (2001), pp. 1485-1489.

5) Laourine E., Schneider, M. and Wulfhorst B.: Production and Analysis of 3D Braided Textile Preforms for Composites, Texcomp 5, Belgium September 2000.

6) Cai, X., Zhou, G. and Liao, D.: Analyses of the Stiffness and Damp- ing of the 4 Step Three Dimensional Braided Composite Links, $J$. Mech. Strength, (1999): 01.

7) Cawley, P., and Adams, R. D. : A Vibration Technique for NonDestructive Testing of Fibre Composite Structures, J. Compos. Mater, 132 (1979), pp. 161-175.

8) Li, D., Lu, Z., Li, J. and Chen, L.: Experimental Research on the Vibration Damping Properties of 3D Braided Composites, J. Mech. Strength, 2009: 02.

9) Pei, X., Li, J., Chen, K., and Ding, G.: Vibration modal analysis of three-dimensional and four directional braided composites.Composites Part B, 69 (2015), pp. 212-221.

10) Pei, X., Chen, L., Li, J., Tang, Y. and Chen, K.: Effect of Damage on the Vibration Modal of a Novel Three-Dimensional and Four-directional Braided Composite T-Beam, Composites Part B, 86 (2016), pp.108-119.

11) ANSYS, Inc., Canonsburg, Pennsylvania, ANSYS Mechanical APDL Modeling and Meshing Guide, Release 15.0, 2013. 